\title{
DIFFERENTIABLE FUNCTIONS DEFINED IN ARBITRARY SUBSETS OF EUCLIDEAN SPACE*
}

\author{
BY \\ HASSLER WHITNEY
}

1. Introduction. In a former paper $\dagger$ we studied the differentiability of a function defined in closed subsets of Euclidean $n$-space $E$. We consider here the differentiability "about" an arbitrary point of a function defined in an arbitrary subset of $E$. We show in Theorem 1 that any function defined in a subset $A$ of $E$ which is differentiable about a subset $B$ of $E$ may be extended over $E$ so that it remains differentiable about $B$. This theorem is a generalization of AE Lemma 2. We show further that any function of class $C^{m}$ about a set $B$ is of class $C^{m-1}$ about an open set $B^{\prime}$ containing $B$. In the second part of the paper we consider some elementary properties of differentiable functions, such as: the sum or product of two such functions is such a function. $¥ \mathrm{We}$ end with the theorem that differentiability is a local property. $\S$

2. Definitions and elementary properties. We use a one-dimensional notation as in AE. Thus $f_{k}(x)=f_{k_{1}} \cdots k_{n}\left(x_{1}, \cdots, x_{n}\right), x^{l}=x_{1}{ }^{l_{1}} \cdots x_{n}{ }^{l_{n}}, l !=l_{1} ! \cdots l_{n} !$, $D_{k} f(x)=\partial^{k_{1}+\cdots+k_{n}} f(x) / \partial x_{1}^{k_{1}} \cdots \partial x_{n}^{k_{n}}$, etc.; we set $\sigma_{k}=k_{1}+\cdots+k_{n}, r_{x y}=$ distance from $x$ to $y$. We always set $f(x)=f_{0}(x)$. Suppose the functions $f_{k}(x)$ for $\sigma_{k} \leqq m$ are defined in the subset $A$ of Euclidean $n$-space $E$. Define $R_{k}\left(x^{\prime} ; x\right)$ for $x, x^{\prime}$ in $A$ by

* Presented to the Society, January 2, 1936; received by the editors October 26, 1935.

$\dagger$ Analytic extensions of differentiable functions defined in closed sets, these Transactions, vol. 36 (1934), pp. 63-89. We refer to this paper as AE. See also Functions differentiable on the boundaries of regions, Annals of Mathematics, vol. 35 (1934), pp. 482-485, and Differentiable functions defined in closed sets, I, these Transactions, vol. 36 (1934), pp. 369-387, which we refer to as F and D respectively.

P. Franklin in Theorem 1 of a paper Derivatives of higher order as single limits, Bulletin of the American Mathematical Society, vol. 41 (1935), pp. 573-582, has given a necessary and sufficient condition for the existence of a continuous $m$ th derivative. We remark that this theorem is exactly the special case of Theorem I of D obtained by letting $f(x)$ be defined in an interval. It is also a special case of Theorem 2 of the author's Derivatives, difference quotients, and Taylor's formula, Bulletin of the American Mathematical Society, vol. 40 (1934), pp. 89-94 (see also Errata, p. 894). For his assumption is easily seen to imply the needed uniformity condition; it also implies at once that $f(x)$ is continuous, so that no considerations of measurability are necessary. His Theorem 2 should be compared with Theorems II and III of D.

$\ddagger$ If the set is closed, these theorems may be proved by first extending the functions throughout $E$.

$\S$ For the case of one variable this follows from D, Theorem I. 


$$
f_{k}\left(x^{\prime}\right)=\sum_{\sigma_{l} \leqq m-\sigma_{k}} \frac{f_{k+l}(x)}{l !}\left(x^{\prime}-x\right)^{l}+R_{k}\left(x^{\prime} ; x\right) .
$$

Let $x^{0}$ be an arbitrary point of $E$. If for each $k\left(\sigma_{k} \leqq m\right)$ and every $\epsilon>0$ there is a $\delta>0$ such that

$$
\left|R_{k}\left(x^{\prime} ; x\right)\right| \leqq r_{x x^{\prime}}^{m-\sigma_{k}} \in \text { if } x, x^{\prime} \text { in } A, r_{x x^{0}}<\delta, r_{x^{\prime} x^{0}}<\delta,
$$

we shall say that $f(x)$ is of class $C^{m}$ in $A$ about $x^{0}$ in terms of the $f_{k}(x)$, or, $f(x)$ is $\left(C^{m}, A, x^{0}, f_{k}(x)\right)$. If this is true for each $x^{0}$ in $B$, we say $f(x)$ is $\left(C^{m}, A, B, f_{k}(x)\right)$, and replace "about $x^{0}$ " by "about $B$." We say $f(x)$ (defined in $A$ ) is of class $C^{m}$ in $A$ about $B$, or, $f(x)$ is $\left(C^{m}, A, B\right)$, if there exist functions $f_{k}(x)\left(\sigma_{k} \leqq m\right)$ defined in $A$ such that $f(x)$ is $\left(C^{m}, A, B, f_{k}(x)\right)$. If $B=A$ in the last two definitions, we leave out the words "about $B$ "; this is in agreement with the previous definitions. We say $f(x)$ is $\left(C^{\infty}, A, B, f_{k}(x)\right)$ if $f(x)$ is $\left(C^{m}, A, B, f_{k}(x)\right)$ for each $m$. Any function defined in $A$ is $\left(C^{-1}, A, E\right)$.

Remark. We might define in an obvious manner such relations as $\left(C^{m}, A, x^{0}\right),\left(C^{\infty}, A, B\right)$. To study them would require a study of the different possible definitions of the $f_{k}(x)$ if $f(x)$ is $\left(C^{m}, A, B\right)$. The $f_{k}(x)$ are not in general determined by $f(x)$. Thus if $A=B$ is the $x_{1}$-axis, only the $f_{k}(x)$ with $k_{2}=\cdots=k_{n}=0$ are determined by $f(x)$. It is not obvious for what point sets $A$ the $f_{k}(x)$ are all determined by $f(x)$.

If $f(x)$ is $\left(C^{m}, A, B, f_{k}(x)\right)(m \geqq 0)$, then the $f_{k}(x)$ are continuous at each point of $B$; that is, the $f_{k}(x)$ may be defined in $B-B \cdot A$ so that this will be true. To show this, take $x^{0}$ in $B$, set $\epsilon=1$, and choose $\delta$ so that (2) holds for any $k\left(\sigma_{k} \leqq m\right)$. Take $x$ in $A$ within $\delta$ of $x^{0}$ (if there is such a point); then (1) and (2) show that $f_{k}\left(x^{\prime}\right)$ is bounded for $x^{\prime}$ in $A$ within $\delta$ of $x^{0}\left(\sigma_{k} \leqq m\right)$. Now let $\left\{x^{i}\right\}$ be any sequence of points of $A, x^{i} \rightarrow x^{0}$; (1) and (2) show that $\left\{f_{k}\left(x^{i}\right)\right\}$ is a regular sequence.

If $A$ is open and $f(x)$ is $\left(C^{m}, A, A, f_{k}(x)\right)$, then $D_{k} f(x)$ exists and equals $f_{k}(x)$ in $A\left(\sigma_{k} \leqq m\right)$. (See AE.) If $x^{0}$ is an isolated point of $A$ or is at a positive distance from $A$, then $f(x)$ is $\left(C^{m}, A, x^{0}, f_{k}(x)\right)$ for any $f_{k}(x)$. If $f(x)$ is $\left(C^{m}, A, B, f_{k}(x)\right)$ [or $\left.\left(C^{m}, A, B\right)\right]$, and $A^{\prime}$ is in $A, B^{\prime}$ is in $B$, then $f(x)$ is $\left(C^{m}, A^{\prime}, B^{\prime}, f_{k}(x)\right)$ [or $\left(C^{m}, A^{\prime}, B^{\prime}\right)$ ]. Also $f(x)$ is $\left(C^{0}, A, B\right)$ if and only if it is continuous at each point of $B$. If $f(x)$ is $\left(C^{m}, A, B, f_{k}(x)\right)$, then it is $\left(C^{m^{\prime}}, A, B, f_{k}(x)\right)$ for all $m^{\prime}<m$; a stronger theorem is proved in Theorem 2 . If $f(x)$ is $\left(C^{m}, A, B, f_{k}(x)\right)$, then $f_{k}(x)$ is $\left(C^{m-\sigma_{k}}, A, B, f_{l}(x)\right)$.

3. Extension theorems. We prove here a theorem which gives the maximum range of differentiability of a function, and a theorem about the still larger range of differentiability of a function to an order one less.

\footnotetext{
* Or better, "continuous in $A$ about $B . "$
} 
THEOREM 1. If $f(x)$ is $\left(C^{m}, A, B, f_{k}(x)\right)^{*}$ ( $m$ finite or infinite), then the $f_{k}(x)$ may be extended throughout $E$ so that $f(x)$ is $\left(C^{m}, E, B, f_{k}(x)\right) . \dagger$

We note, conversely, that if $f(x)$ is not $\left(C^{m}, A, x^{0}, f_{k}(x)\right)$, then no extension of $f(x)$ will be so. We remark also that $f(x)$ may be made analytic in $E-\bar{A}$ $(\bar{A}=A$ plus limit points).

To prove the theorem, we first extend the $f_{k}(x)$ through $\bar{A}-A$ as follows: Take any $x^{0}$ in $\bar{A}-A$. Let $f_{k}\left(x^{0}\right)$ be the upper limit of $f_{k}\left(x^{i}\right)$ for sequences $\left\{x^{i}\right\}, x^{i} \rightarrow x^{0}, x^{i}$ in $A$, if this is finite; otherwise, set $f_{k}\left(x^{0}\right)=0$. Next we extend the $f_{k}(x)$ throughout $E-\bar{A}$ by the method of AE Lemma 2. We shall assume in the proof that $m$ is finite. If $m=\infty$, we prove $C^{m^{\prime}}$ for every integer $m^{\prime}$. The only alteration needed in the proof is that AE $\$ 12$ should be used; but this makes no essential change.

As $E-\bar{A}$ is open, $f(x)$ is $\left(C^{m}, E, E-\bar{A}, f_{k}(x)\right)$; we must show that $f(x)$ is $\left(C^{m}, E, B \cdot \bar{A}, f_{k}(x)\right)$. Take a fixed point $x^{0}$ in $B \cdot \bar{A}$. Let us say $\left(k, \epsilon, A_{1}, A_{2}\right)$ holds if there is a $\delta>0$ such that (2) holds whenever $x$ is in $A_{1}, x^{\prime}$ is in $A_{2}$, and $r_{x x^{0}}<\delta, r_{x^{\prime} x^{0}}<\delta$. We must prove $(k, \epsilon, E, E)$ for each $k\left(\sigma_{k} \leqq m\right)$ and each $\epsilon>0$.

First we prove $(k, \epsilon, \bar{A}, \bar{A})$. Set $\epsilon^{\prime}=\epsilon /\left[2(m+1)^{n}\right]$, and let $\delta$ be the smallest of the $\delta$ 's given by $\left(l, \epsilon^{\prime}, A, A\right)$ for $\sigma_{l} \leqq m$. Let $U^{\prime}$ be the spherical neighborhood of $x^{0}$ of radius $\delta$; then $f_{l}(x)$ is bounded in $U \cdot A\left(\sigma_{l} \leqq m\right)$. Given $x, x^{\prime}$ in $U \cdot \bar{A}$, choose sequences $\left\{x^{i}\right\},\left\{x^{\prime i}\right\}$ of points of $U \cdot A$, with $x^{i} \rightarrow x, x^{\prime i} \rightarrow x^{\prime}$. Suppose first $\sigma_{k}=m$. Then we may take these sequences so that $f_{k}\left(x^{i}\right) \rightarrow f_{k}(x)$, $f_{k}\left(x^{\prime}\right) \rightarrow f_{k}\left(x^{\prime}\right)$, and the desired inequality for $R_{k}\left(x^{\prime} ; x\right)$ follows from that for $R_{k}\left(x^{\prime i} ; x^{i}\right)$. Suppose now that $\sigma_{k}<\dot{m}$. Relations (1) and (2) with $k, x^{\prime}$, $x$ replaced by $l, x^{i}, x^{j}$ show that for any such $\left\{x^{i}\right\},\left\{f_{l}\left(x^{i}\right)\right\}$ is a regular sequence $\left(\sigma_{l}<m\right)$; hence $f_{l}\left(x^{i}\right) \rightarrow f_{l}(x)$, and similarly $f_{l}\left(x^{\prime}\right) \rightarrow f_{l}\left(x^{\prime}\right) \quad\left(\sigma_{l}<m\right)$. Relation (1) now shows that for $i$ large enough, $\Delta=R_{k}\left(x^{\prime} ; x\right)-R_{k}\left(x^{\prime i} ; x^{i}\right)$ differs as little as we please from

$$
-\sum_{\sigma_{l}=m-\sigma_{k}} \frac{\dot{f}_{k+l}(x)-f_{k+l}\left(x^{i}\right)}{l !}\left(x^{\prime}-x\right)^{l} .
$$

As $\left|f_{j}(x)-f_{j}\left(x^{i}\right)\right| \leqq \epsilon^{\prime}\left(\sigma_{j}=m\right)$ and $\left|\left(x^{\prime}-x\right)^{l}\right| \leqq r_{x x^{\prime}}^{\sigma_{l}},|\Delta| \leqq(m+1)^{n} \epsilon^{\prime} r_{x x^{\prime}}^{m-\sigma_{k}}$ for $i$ large enough; the inequality again follows.

Next we prove $(k, \epsilon, \bar{A}, E-\bar{A})$. Set $\epsilon^{\prime}=\epsilon /\left[2 \cdot 4^{m}(m+1)^{n}\right]$, and define $\eta$ in terms of $\epsilon^{\prime}$ and then $\delta$ as in $\mathrm{AE} \$ 11$, using $(k, \eta, \bar{A}, \bar{A})$. Take $x$ in $\bar{A}$ and $x^{\prime}$ in $E-\bar{A}$, each within $\delta / 4$ of $x^{0}$. By $\operatorname{AE}(6.3)$ and the equation following (11.6),

* Or merely locally $\left(C^{m}, A, B\right)$; see Theorem 6 .

† If $A=B$ is closed, then $B$ may be replaced by $E$; the present proof then gives a proof of $\mathrm{AE}$ Lemma 2 which makes no use of AE Lemma 1. 


$$
\begin{aligned}
R_{k}\left(x^{\prime} ; x\right) & =D_{k} f\left(x^{\prime}\right)-\psi_{k}\left(x^{\prime} ; x\right) \\
& =\sum_{l} \frac{R_{k+l}\left(x^{*} ; x\right)}{l !}\left(x^{\prime}-x^{*}\right)^{l}+\sum_{s=1}^{t} \sum_{l}\left(\begin{array}{l}
k \\
l
\end{array}\right) D_{l} \phi_{\lambda_{s}}\left(x^{\prime}\right) \zeta_{\lambda_{s} ; k-l}\left(x^{\prime}\right),
\end{aligned}
$$

where $x^{*}$ is a point of $\bar{A}$ distant $\delta_{*} / 4$ from $x^{\prime}, \delta_{*} / 4$ being the distance from $x^{\prime}$ to $\bar{A}$. As $r_{x^{*} x} \leqq 2 r_{x x^{\prime}}, r_{x^{\prime} x^{*}} \leqq 2 r_{x x^{\prime}}$, and $\delta_{*} \leqq 4 r_{x x^{\prime}}$, we find with the help of $\mathrm{AE}(11.8)$

$$
\left|R_{k}\left(x^{\prime} ; x\right)\right| \leqq(m+1)^{n}\left(2 r_{x x^{\prime}}\right)^{m-\sigma_{k}} \eta+\left(4 r_{x x^{\prime}}\right)^{m-\sigma_{k}} \epsilon^{\prime} / 2<r_{x x^{\prime}}^{m-\sigma_{k}} \epsilon .
$$

Next we prove $(k, \epsilon, E-\bar{A}, \bar{A})$. As is easily seen from AE (6.3) or by $\mathrm{F}$ (6) with $x^{i-1}, x^{i}$ replaced by $x, x^{\prime}$,

$$
R_{k}\left(x^{\prime} ; x\right)=\sum_{l} \frac{R_{k+l}\left(x ; x^{\prime}\right)}{l !}\left(x^{\prime}-x\right)^{l} .
$$

Set $\epsilon^{\prime}=\epsilon /(m+1)^{n}$, and take the smallest $\delta$ given by $\left(k+l, \epsilon^{\prime}, \bar{A}, E-\bar{A}\right)$ for $\sigma_{l} \leqq m-\sigma_{k}$. The required inequality now follows at once.

Finally we must show $(k, \epsilon, E-\bar{A}, E-\bar{A})$. Set $\epsilon^{\prime}=\epsilon /\left[2 n(m+1)^{n}\right]$, and take $\delta$ smaller than the $\delta / 4$ given by AE $\$ 11$ with $\epsilon$ replaced by $\epsilon^{\prime}$ and smaller than the $\delta$ 's given by $\left(k+l, \epsilon^{\prime}, \bar{A}, E-\bar{A}\right)$ and $\left(k+l, \epsilon^{\prime}, E-\bar{A}, \bar{A}\right)$ for $\sigma_{l} \leqq m-\sigma_{k}$. Now take $x$ and $x^{\prime}$ in $E-\bar{A}$ within $\delta$ of $x^{0}$; we must consider two cases. Case I: The line segment $S=x x^{\prime}$ lies wholly in $E-\bar{A}$. By AE (11.2), $\left|f_{l}(y)-f_{l}\left(x^{\prime}\right)\right|<2 \epsilon^{\prime}$ for $y$ on $S\left(\sigma_{l} \leqq m\right)$; the desired inequality now follows from F, Lemma 3. Case II: There is a point $x^{*}$ of $\bar{A}$ on $S$. From AE (6.3), or $\mathrm{F}(6)$ with $x^{i-1}, x^{i}$ replaced by $x, x^{*}$, we find

$$
R_{k}\left(x^{\prime} ; x\right)=R_{k}\left(x^{\prime} ; x^{*}\right)+\sum_{l} \frac{R_{k+l}\left(x^{*} ; x\right)}{l !}\left(x^{\prime}-x^{*}\right)^{l},
$$

and the inequality again follows.

THEOREM 2. If $f(x)$ is ( $C^{m}, A, B, f_{k}(x)$ ) ( $m$ finite), then there is an open set $B^{\prime}$ containing $B$ such that $f(x)$ is $\left(C^{m-1}, A, B^{\prime}, f_{k}(x)\right)$.

For each $x$ in $B$, let $\delta(x)$ be the largest of the numbers $\delta$ for which (2) holds for all $k\left(\sigma_{k} \leqq m\right)$ with $\epsilon$ replaced by 1 . Let $U(x)$ be the set of all points $x^{\prime}$ within $\delta(x)$ of $x$; then $B^{\prime}$ is the sum of all $U(x)$. The set $B^{\prime}$ is open. To prove $\left(C^{m-1}, A, B^{\prime}, f_{k}(x)\right)$, take any $x^{0}$ in $B^{\prime}$ and any $\epsilon>0$. For some $x^{*}$ in $B$, $r_{x^{*} x^{0}}<\delta\left(x^{*}\right)$. There is an $M$ such that $\left|f_{k}(y)\right|<M$ for $y$ in $A \cdot U\left(x^{*}\right)\left(\sigma_{k} \leqq m\right)$. $\dagger$ Let $\delta$ be the smaller of $\delta\left(x^{*}\right)-r_{x^{*} x^{0}}$ and $\epsilon /\left[2(m+1)^{n} M+2\right]$. Now take any $x$ and $x^{\prime}$ in $A$ within $\delta$ of $x^{0}$. We are interested in the remainders

$\dagger$ For the proof, see the paragraph following the remark. 


$$
R_{k}^{\prime}\left(x^{\prime} ; x\right)=\sum_{\sigma_{l}=m-\sigma_{k}} \frac{f_{k+l}(x)}{l !}\left(x^{\prime}-x\right)^{l}+R_{k}\left(x^{\prime} ; x\right)
$$

with $\sigma_{k}<m$. As $r_{x x^{\prime}}<2 \delta$,

$$
\left|R_{k}^{\prime}\left(x^{\prime} ; x\right)\right| \leqq(m+1)^{n} M r_{x x^{\prime}}^{m-\sigma_{k}}+r_{x x^{\prime}}^{m-\sigma_{k}}<r_{x x^{\prime}}^{m-1-\sigma_{k}} \epsilon .
$$

Corollary. If $f(x)$ is of class $C^{m}$ in any given point set about $B$, then it may be extended through an open set $B^{\prime}$ containing $B$ so that it is of class $C^{m-1}$ in $B^{\prime}$ and of class $C^{m}$ in $B^{\prime}$ about $B$.

4. Composite functions, etc. We prove here three theorems.

Theorem 3. If $f$ and $g$ are of class $C^{m}$ in $A$ about $B$, then so are $f+g$ and $f-g$, with

$$
(f \pm g)_{k}=f_{k} \pm g_{k}
$$

This is obvious.

Theorem 4. If $f$ and $g$ are of class $C^{m}$ in $A$ about $B$, then so is $f g$, and $f / g$ if $g \neq 0$. The derivatives are given by the ordinary formulas. Thus

$$
(f g)_{k}=\sum_{l}\left(\begin{array}{l}
k \\
l
\end{array}\right) f_{l} g_{k-l} .
$$

We might prove this theorem directly, but it follows from Theorem 5: $f g$ and $f / g$ are functions (of two variables) of class $C^{\infty}$ of the functions $f$ and $g$. (The condition $B$ in $A$ is obtained by using Theorem 1.)

Theorem 5. Let $A$ and $B$ be subsets of $n$-space $E_{n}$, and let $A^{\prime}$ and $B^{\prime}$ be subsets of $\nu$-space $E_{\nu}$. Let $f^{i}(x)$ be $\left(C^{m}, A, B, f_{k}^{i}(x)\right)(i=1, \cdots, \nu)$, and let $g(y)$ be $\left(C^{m}, A^{\prime}, B^{\prime}, g_{k}(y)\right.$ ) (m finite or infinite). Suppose $B$ is in $A, x$ in $A$ implies

$$
y=\left(y_{1}, \cdots, y_{v}\right)=\left(f^{1}(x), \cdots, f^{\nu}(x)\right)=f(x)
$$

in $A^{\prime}$, and $x$ in $B$ implies $f(x)$ in $B^{\prime}$. Then the function

$$
h(x)=g\left(f^{1}(x), \cdots, f^{\nu}(x)\right)=g(f(x))
$$

is $\left(C^{m}, A, B, h_{k}(x)\right)$; the $h_{k}(x)$ are given by the ordinary formulas (9) for derivatives.

As a consequence of this theorem, the definition of being of class $C^{m}$ is independent of the coordinate system chosen. If the condition $x$ in $A$ [or $B$ ] does not imply $f(x)$ in $A^{\prime}$ [or $B^{\prime}$ ], we may apply the theorem to any subset $A_{1}$ [or $B_{1}$ ] of $A$ [or $B$ ] for which it does. We shall suppose $m$ is finite; if $m=\infty$, we merely apply the reasoning below for each positive integer. 
Suppose first $u^{1}(x), \cdots, u^{\nu}(x)$ are functions of class $C^{m}$ in an open set $\Gamma$ of $E_{n}$, suppose $v(y)$ is of class $C^{m}$ in an open set $\Gamma^{\prime}$ of $E_{\nu}$, and suppose $x$ in $\Gamma$ implies $u(x)$ in $\Gamma^{\prime}$. Letting $R^{\prime i}, S^{\prime}$ denote remainders for $u^{i}$, v, Taylor's formula gives

$$
\begin{gathered}
u_{k}^{i}\left(x^{\prime}\right)=D_{k} u^{i}\left(x^{\prime}\right)=\sum_{\sigma_{l} \leqq m-\sigma_{k}} \frac{u_{k+l}^{i}(x)}{l !}\left(x^{\prime}-x\right)^{l}+R_{k}^{\prime i}\left(x^{\prime} ; x\right), \\
v_{k}\left(y^{\prime}\right)=D_{k} v\left(y^{\prime}\right)=\sum_{\sigma_{l}^{\prime} \leqq m-\sigma_{k}^{\prime}} \frac{v_{k+l}(y)}{l !}\left(y^{\prime}-y\right)^{l}+S_{k}^{\prime}\left(y^{\prime} ; y\right),
\end{gathered}
$$

certain inequalities on the $R_{k}^{\prime i}$ and $S_{k}^{\prime}$ being satisfied. We have set $\sigma_{k}^{\prime}=k_{1}+\cdots+k_{\nu}$. Set $w(x)=v(u(x))$; then (5) and (6) with $k=0$ give

$$
\begin{aligned}
w\left(x^{\prime}\right)=\sum_{t} \frac{v_{t}(u(x))}{t !}\left\{\sum_{\sigma_{j} \geqq 1} \frac{u_{j}(x)}{j !}\left(x^{\prime}-x\right)^{j}+R^{\prime}\left(x^{\prime} ; x\right)\right\}^{t} & \\
& +S^{\prime}\left(u\left(x^{\prime}\right) ; u(x)\right),
\end{aligned}
$$

where $S^{\prime}=S_{0}^{\prime}$. Also, by Taylor's formula,

$$
w_{k}\left(x^{\prime}\right)=\sum_{l} \frac{w_{k+l}(x)}{l !}\left(x^{\prime}-x\right)^{l}+T_{k}^{\prime}\left(x^{\prime} ; x\right) .
$$

Subtract (8) with $k=0$ from (7); then as $R^{\prime i}, S^{\prime}$, and $T^{\prime}$ all approach 0 to the $m$ th order as $x^{\prime} \rightarrow x, \dagger$ we may equate coefficients of $\left(x^{\prime}-x\right)^{k}$ for $\sigma_{k} \leqq m . \ddagger$ Thus we find polynomials

$$
P_{k}\left(u_{p}^{i}, v_{q}\right) \quad\left(\sigma_{p} \leqq \sigma_{k}, \sigma_{q}^{\prime} \leqq \sigma_{k} ; \sigma_{k} \leqq m\right)
$$

such that, for any $x$ in $\Gamma$,

$$
w_{k}(x)=P_{k}\left(u_{p}^{i}(x), v_{q}(u(x))\right) .
$$

Using (8) gives for $w_{k}\left(x^{\prime}\right)$

$$
w_{k}\left(\dot{x}^{\prime}\right)=\sum_{l} \frac{P_{k+l}\left(u_{p}^{i}(x), v_{q}(u(x))\right)}{l !}\left(x^{\prime}-x\right)^{l}+T_{k}^{\prime}\left(x^{\prime} ; x\right) .
$$

We may also evaluate it by replacing $x$ by $x^{\prime}$ in (9) and using (5) and (6). (In (6) we replace $y^{\prime}$ by $u\left(x^{\prime}\right)$ and use (5) again.) Each variable in the resulting polynomial $P_{k}$ consists of a polynomial in quantities $R^{\prime}, S^{\prime}$, and other quantities; if we multiply out and collect all terms with an $R^{\prime}$ or an $S^{\prime}$ as a factor, we obtain

$\dagger$ This is clear for $S^{\prime}$ if $m=0$; if $m>0$, then

$$
S^{\prime} / r_{x x^{\prime}}^{m}=\left[S^{\prime} /\left|u\left(x^{\prime}\right)-u(x)\right|^{m}\right] \cdot\left[\left|u\left(x^{\prime}\right)-u(x)\right| / r_{x x^{\prime}}\right]^{m},
$$

where $\left|y^{\prime}-y\right|=r_{y y^{\prime}}$, and the last factor is bounded in $U \cdot A$.

$\ddagger$ This is easily proved in succession for $\sigma_{k}=0,1, \cdots$ on letting $x^{\prime} \rightarrow x$. 


$$
\begin{aligned}
& w_{k}\left(x^{\prime}\right)=P_{k}\left[\sum_{s} \frac{u_{p^{i}+s}(x)}{s !}\left(x^{\prime}-x\right)^{s},\right. \\
& \left.\sum_{t} \frac{v_{q+t}(u(x))}{t !}\left\{\sum_{\sigma_{j} \geqq 1} \frac{u_{j}(x)}{j !}\left(x^{\prime}-x\right)^{j}\right\}^{t}\right]+Q_{k},
\end{aligned}
$$

where $Q_{k}$ is a polynomial containing an $R^{\prime}$ or an $S^{\prime}$ as a factor in each term. It must be understood that $\sum u_{p+s}^{i}(x)\left(x^{\prime}-x\right)^{s} / s !$ appears as the variable in the position of $u_{p}^{i}$, etc., in $P_{k}\left(u_{p}^{i}, v_{q}\right)$.

We now prove: If $u_{k}^{i}\left(\sigma_{k} \leqq m ; i=1, \cdots, \nu\right), v_{k}\left(\sigma_{k}^{\prime} \leqq m\right)$ are any numbers, then

$$
\begin{aligned}
P_{k}^{*}\left(x ; u_{p}^{i}, v_{q}\right)=P_{k}\left[\sum_{s} \frac{u_{p+s}^{i}}{s !} x^{s}, \sum_{t} \frac{v_{q+t}}{t !}\left\{\sum_{\sigma j \geqq 1} \frac{u_{j}}{j !} x^{j}\right\}^{t}\right] \\
-\sum_{l} \frac{P_{k+l}\left(u_{p}^{i}, v_{q}\right)}{l !} x^{l},
\end{aligned}
$$

considered as a polynomial in $x$, contains no terms of degree $\leqq m-\sigma_{k}$. To prove this, define the polynomials

$$
u^{i}(x)=\sum_{\sigma_{l} \leqq m} \frac{u_{l}^{i}}{l !} x^{l}, \quad v(y)=\sum_{\sigma_{l}^{\prime} \leqq m} \frac{v_{l}}{l !}\left(y-u_{0}\right)^{l} ;
$$

then $u_{k}^{i}(0)=D_{k} u^{i}(0)=u_{k}^{i}, v_{k}\left(u_{0}\right)=D_{k} v\left(u_{0}\right)=v_{k}$. Set $w(x)=v(u(x))$. Replacing $x^{\prime}, x$ by $x, 0$ in (10) and (11) and putting in (12) gives, as $Q_{k}=0$ in this case,

$$
P_{k}^{*}\left(x ; u_{p}^{i}, v_{q}\right)=T_{k}^{\prime}(x ; 0) .
$$

As $T_{k}^{\prime} \rightarrow 0$ to the $\left(m-\sigma_{k}\right)$ th order as $x \rightarrow 0, P_{k}^{*}$ cannot contain any terms of degree $\leqq m-\sigma_{k}$.

We return now to the functions $f^{i}(x), g(y), h(x)$. Set $h_{k}(x)=P_{k}\left(f_{p}^{i}(x)\right.$, $\left.g_{q}(f(x))\right)$. The formulas (10) and (11) hold equally well for the $f^{i}, g, h$. Hence using (10), (11), and (12), we find for the remainder for $h_{k}(x)$

$$
T_{k}\left(x^{\prime} ; x\right)=P_{k}^{*}\left(x^{\prime}-x ; f_{p}^{i}(x), g_{q}(f(x))\right)+Q_{k} .
$$

To show that $h(x)$ is $\left(C^{m}, A, B, h_{k}(x)\right)$, take any $x^{0}$ in $B$, and set $y^{0}=f\left(x^{0}\right)$. As $f(x)$ is continuous in $A$ about $B$, for each neighborhood $V$ of $y^{0}$ there is a neighborhood $U(V)$ of $x^{0}$ such that $x$ in $U(V) \cdot A$ implies $f(x)$ in $V \cdot A^{\prime}$. As $y^{0}$ is in $B^{\prime}$, we may take $V$ so that the $g_{k}(y)$ are bounded in $V \cdot A^{\prime}$. We may take $U$ in $U(V)$ so small that the $f_{k}(x)$ are bounded in $U \cdot A$. Because of the property of $P_{k}^{*}$, we may obviously take $\delta$ small enough so that $P_{k}^{*}$ satisfies an inequality of the nature of (2). Moreover each term in $Q_{k}$ contains an $R_{p}\left(x^{\prime} ; x\right)$ or an $S_{q}\left(u\left(x^{\prime}\right) ; u(x)\right)$ with $\sigma_{p} \leqq \sigma_{k}$ or $\sigma_{q}^{\prime} \leqq \sigma_{k}$; as each such remainder satisfies 
an inequality (2) (see a recent footnote) and all other quantities entering into $Q_{k}$ are bounded, we may take $\delta$ small enough so that $Q_{k}$ also satisfies an inequality (2). Hence the same is true of $T_{k}$, and the theorem is proved.

5. Differentiability a local property. Our object is to prove

Theorem 6. Let $f(x)$ be locally $\left(C^{m}, A, B\right)$ ( $m$ finite or infinite). For each point $x^{0}$ of $B$ there is a neighborhood $U$ of $x^{0}$ and functions $f_{k}^{\left(x^{0}\right)}(x)$ defined in $U \cdot A$ such that $f(x)$ is $\left(C^{m}, U \cdot A, U \cdot B, f_{k}\left(x^{0}\right)(x)\right)$. + Then $f(x)$ is $\left(C^{m}, A, B\right)$. If the $f_{k}{ }^{\left(x^{0}\right)}(x)$ for $\sigma_{k} \leqq p$ are independent (at any $x$ for which they are defined) of $x^{0}$, then these functions may be included among the $f_{k}(x)\left(\sigma_{k} \leqq m\right)$.

We may take each neighborhood $U$ as an open $n$-cube, so small that the $f_{k}{ }^{\left(x^{0}\right)}(x)$ are bounded in $U$. A finite or denumerable number of them, $C_{1}, C_{2}, \cdots$, cover $B$; we may take them so that any one touches at most a finite number of the others, and so that any boundary point of any $C_{i}$ is interior to some $C_{j} . \ddagger$ By hypothesis, to each $i$ there correspond functions $f_{k}^{i}(x)$, $\sigma_{k} \leqq m$, such that $f(x)$ is $\left(C^{m}, C_{i} \cdot A, C_{i} \cdot B, f_{k}^{i}(x)\right)$. In each $C_{i}$ we define the function $\pi_{i}(x)$ as it was defined in $I_{i}$ in $\mathrm{AE} \S 9$; set

$$
\phi_{i}(x)=\pi_{i}(x) / \sum_{j} \pi_{j}(x)
$$

in $C_{1}+C_{2}+\cdots$. Set $g^{i}(x)=\phi_{i}(x) f(x)$ in $C_{i} \cdot A$. By Theorem $4, g^{i}(x)$ is $\left(C^{m}, C_{i} \cdot A, C_{i} \cdot B\right)$, and

$$
g_{k}^{i}(x)=\sum_{l}\left(\begin{array}{l}
k \\
l
\end{array}\right) D_{l} \phi_{i}(x) f_{k-l}^{i}(x) .
$$

As the $f_{k}^{i}(x)$ are bounded in $C_{i} \cdot A$ and the $D_{l} \phi_{i}(x) \rightarrow 0$ to infinite order as $x$ approaches the boundary of $C_{i}$ (see $\mathrm{AE} \S 9$ ), the latter statement is true also of the $g_{k}^{i}(x)$. Hence, evidently, if we set $g_{k}^{i}(x)=0$ in $A-C_{i} \cdot A, g^{i}(x)$ is $\left(C^{m}, A, B, g_{k}^{i}(x)\right)$. Set

$$
f_{k}(x)=g_{k}^{1}(x)+g_{k}^{2}(x)+\cdots,
$$

which in any $C_{i} \cdot A$ is a finite sum; this reduces to $f(x)$ for $k=0$. Theorem 3 shows at once that $f(x)$ is $\left(C^{m}, A, B, f_{k}(x)\right)$. (Given $x^{0}$ in $B$, to apply Theorem

† Note that " $f(x)$ is locally $\left(C^{\infty}, \cdots\right)$ " is not the same statement as " $f(x)$ is locally $\left(C^{m}, \cdots\right)$ for each $m$."

$\ddagger$ Let $C^{1}, C^{2}, \cdots$ be a denumerable set of the cubes which cover $B$. Express each $C^{i}$ as the sum of a denumerable number of cubes $C_{j}{ }^{i}$ with the following properties: Each $C_{j}{ }^{i}$ is, with its boundary, interior to $C^{i}$; the diameter of $C_{j}{ }^{i}, \delta\left(C_{j}{ }^{i}\right)$, is $\left\langle 1 / i ; \delta\left(C_{j}{ }^{i}\right) \rightarrow 0\right.$ as $j \rightarrow \infty$; the cubes $C_{j}{ }^{i}$ approach the boundary of $C^{i}$ as $j \rightarrow \infty$. Now drop out all cubes $C_{j}{ }^{i}$ which are interior to larger cubes $C_{l}{ }^{k} ;$ the remaining cubes $C_{1}, C_{2}, \cdots$ still cover $B$. To each cube $C_{j}{ }^{i}$ corresponds a number $\eta>0$ such that any point set of diameter $<\eta$ having points in common with $C_{j}{ }^{i}$ lies interior to some $C_{k}{ }^{i}$; using this fact, it is easily seen that any $C_{\boldsymbol{i}}$ has points in common with but a finite number of the $C_{j}$. 
3 , we choose $\delta$ so small that the points within $\delta$ of $x^{0}$ lie in but a finite number of the $C_{i}$.)

To prove the second statement, let $f_{i}^{\prime}(x)$ denote the common value of $f_{l}{ }^{i}(x)$ for $\sigma_{l} \leqq p$. Differentiating $\sum \phi_{i}=1$ gives

$$
\sum_{i} D_{l} \phi_{i}(x)= \begin{cases}1 & \text { if } l=0 \\ 0 & \text { if } \sigma_{l}>0\end{cases}
$$

Define the $f_{k}(x)$ as before. Take any $k$ with $\sigma_{k} \leqq p$; then (17) and (18) give

$$
f_{k}(x)=\sum_{l}\left(\begin{array}{l}
k \\
l
\end{array}\right) f_{k-l}^{\prime}(x) \sum_{i} D_{l} \phi_{i}(x)=f_{k}^{\prime}(x)
$$

in $C_{1}+C_{2}+\cdots$. It does not matter how $f_{k}(x)$ is defined outside this set.

The second statement in the theorem does not hold for an arbitrary set of $f_{k}(x)$, at least using the above method. To see this, take $n=m=2, A=B$ $=$ the interval $(-1,1)$ of the $x_{1}$-axis, $C_{1}=C_{2}=$ the square with corners $( \pm 1, \pm 1)$; set $f=0$,

$$
f_{10}^{1}=f_{20}^{1}=f_{11}^{1}=f_{02}^{1}=0, \quad f_{01}^{1}=1,
$$

and $f_{i j}^{2}=-f_{i j}^{1}$ on $A$. Also set

$$
\phi_{1}(x, y)=\frac{1}{2}+\frac{3}{4} x-\frac{1}{4} x^{3}, \quad \phi_{2}(x, y)=\frac{1}{2}-\frac{3}{4} x+\frac{1}{4} x^{3} .
$$

(Though $\phi_{1}$ and $\phi_{2}$ are not the functions defined above, they have the necessary properties.) We find on $A$

$$
g_{11}^{1}(x, y)=g_{11}^{2}(x, y)=\frac{3}{4}-\frac{3}{4} x^{2}, \quad f_{11}(x, y)=\frac{3}{2}-\frac{3}{2} x^{2} \not \equiv 0 .
$$

HARVARD UNIVERSITY,

Cambridge, Mass. 